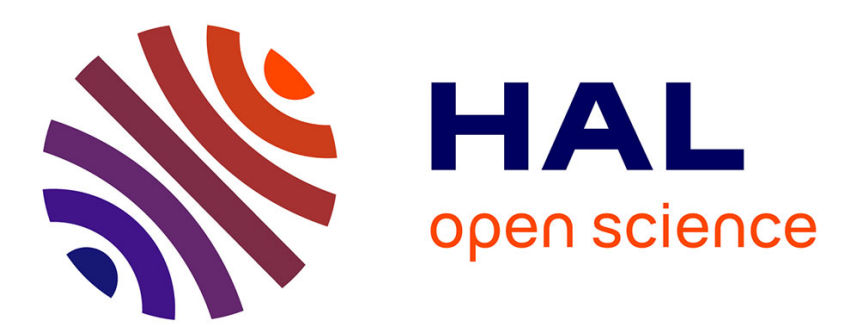

\title{
Some properties of the Irvine cable model and their use for the kinematic analysis of cable-driven parallel robots
} Jean-Pierre Merlet

\section{To cite this version:}

Jean-Pierre Merlet. Some properties of the Irvine cable model and their use for the kinematic analysis of cable-driven parallel robots. Mechanism and Machine Theory, 2019, 135, pp.271 - 280. 10.1016/j.mechmachtheory.2019.02.009 . hal-02426393

\section{HAL Id: hal-02426393 \\ https://hal.inria.fr/hal-02426393}

Submitted on 6 Jan 2020

HAL is a multi-disciplinary open access archive for the deposit and dissemination of scientific research documents, whether they are published or not. The documents may come from teaching and research institutions in France or abroad, or from public or private research centers.
L'archive ouverte pluridisciplinaire HAL, est destinée au dépôt et à la diffusion de documents scientifiques de niveau recherche, publiés ou non, émanant des établissements d'enseignement et de recherche français ou étrangers, des laboratoires publics ou privés. 


\title{
Some properties of the Irvine cable model and their use for the kinematic analysis of cable-driven parallel robots
}

\author{
J-P. Merlet \\ HEPHAISTOS project, Université Côte d'Azur, Inria, France
}

\begin{abstract}
Cable model has a strong influence on the complexity of the kinematic analysis of cable-driven parallel robots (CDPR). The most complete elasto-static model relies on Irvine equation that takes into account both the elasticity and the deformation of the cable due to its own mass and has been shown to be very realistic. This model is complex, non algebraic and numerically ill-conditioned, thereby leading to difficulties when using it in a kinematic analysis involving several cables. We exhibit some properties of this model that may drastically improve the analysis computation time when used in kinematic studies.
\end{abstract}

Keywords: cable-driven parallel robots, cable model, sagging cables

\section{Introduction}

Cables are essential elements in cable-driven parallel robots (CDPR) in which grounded winches independently pay off and reel in cables wound on a drum and attached to a moving platform at the other end. Such a robot has the advantages of parallel robots (accuracy, high velocity, large payload) but also may exhibit large workspace as illustrated by the FAST telescope robot [1], the COGIRO robot of Tecnalia/LIRMM [2] and our MARIONETCRANE prototype [3] (figure 1).

Most of the works related to CDPR assume ideal cables without elasticity and deformation due to the cable mass. With that model the distance $L_{r}$ between the attachment point $B$ of the cable on the CDPR platform and the winch output point $A$ is exactly the paid off cable length $L_{0}$ as measured. However the no-elasticity assumption does not hold for large CDPR with a difference between $L_{0}, L_{r}$ of several centimeters or a variation evaluated at 

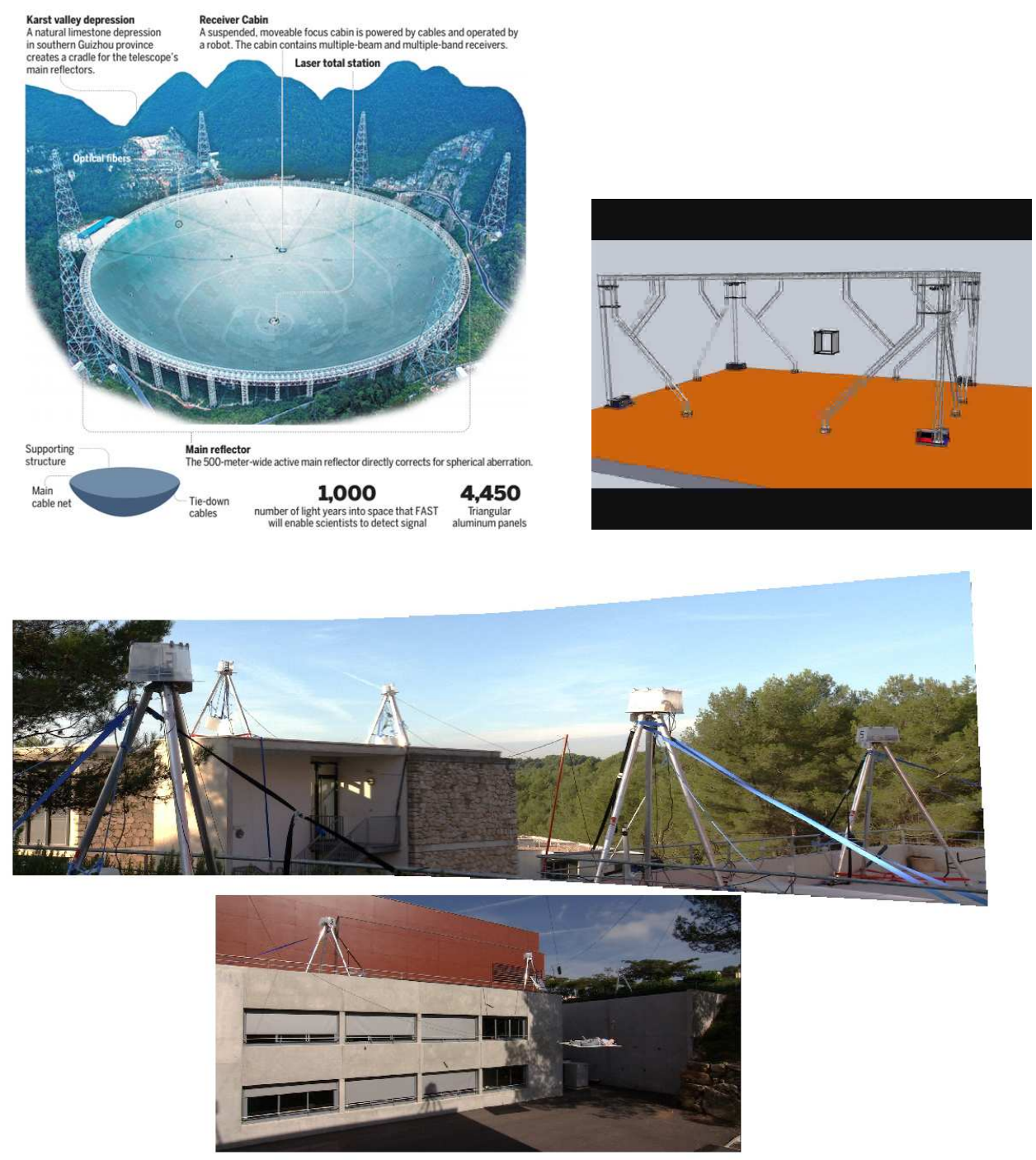

Figure 1: Large CDPR: the FAST telescope, the COGIRO robot and our MARIONETCRANE prototype. The sagging effect on the later CDPR may easily be seen in the video part of the Hephaistos web site. 
$1 \%$ for a small CDPR [4]). As we will see the deformation of the cable due to its own mass induces also significant changes (see figure 3). Neglecting these effects will incur significant errors on the positioning of the CDPR but also on other state variables of the robot such as cable tensions or velocities.

In this paper we will consider the elasto-static Irvine sagging cable model that has been proposed for elastic and deformable cable with mass [5] and that has been shown to be in very good agreement with experimental results [6]. This model assumes that the cable lies in a vertical plane, the cable plane, and is therefore a 2D model. There are also other models that take into account torsion, out-of-plane motion $[7,8]$, the multi-strand nature of the cable [9] or specific to synthetic rope $[9,10,11,12,13]$ but they are mostly valid for cables with a much larger diameter than the one used for CDPR or for cables having a very specific structure. Lumped-mass model have also been proposed [14] but we will see that they are difficult to use for CDPR.

A global reference frame $O,\left(x_{r}, y_{r}, z_{r}\right)$ is defined, with $z$ corresponding to the upward local vertical, and a cable reference frame $A_{i},\left(x, z=z_{r}\right)$ is defined in this plane with its origin at $A_{i}$, one of the extremity of the cable and $x$ in the cable plane, being perpendicular to $z=z_{r}$. The coordinates of the other cable extremity $B_{i}$ are $\left(x_{b} \geq 0, z_{b}\right)$ and we will assume that $B_{i}$ is below $A_{i}$ so that $z_{b} \leq 0$ (Assumption 1). Vertical and horizontal forces $F_{z}, F_{x}>0$ are exerted by the platform on the cable at point $B_{i}$ (figure 2).

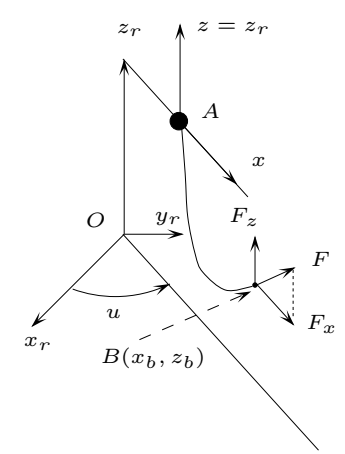

Figure 2: Notation for a sagging cable

Note that $F_{z}$ is negative if the platform is exerting a downward force at $B$ (the tangent of the cable at $B$ has a negative $z$ component), while $F_{z}$ is positive of the tangent of the cable at $B$ has a positive $z$ component. 
For a cable with length at rest $L_{0}$ the coordinates of $B$ are given by the Irvine equations [5]:

$$
\begin{gathered}
x_{b}=F_{x}\left(\frac{L_{0}}{E A_{0}}+\frac{\sinh ^{-1}\left(\frac{F_{z}}{F_{x}}\right)-\sinh ^{-1}\left(\frac{F_{z}-\mu g L_{0}}{F_{x}}\right)}{\mu g}\right) \\
z_{b}=\frac{F_{z} L_{0}-\mu g L_{0}^{2} / 2}{E A_{0}}+\frac{\sqrt{F_{x}^{2}+F_{z}^{2}}-\sqrt{F_{x}^{2}+\left(F_{z}-\mu g L_{0}\right)^{2}}}{\mu g}
\end{gathered}
$$

where $E$ is the Young modulus of the cable material, $A_{0}$ the cable crosssection area and $\mu$ the cable linear density. For example we may illustrate the influence of the cable deformation for a steel cable of diameter $6 \mathrm{~mm}$, length $L_{0}=50$ meters with infinite $E$ (and therefore no elasticity) and $\mu=0.346 \mathrm{~kg} / \mathrm{m}$ under a tension of $100 \mathrm{~N}$ by plotting the difference $L_{0}-L_{r}$ as a function of $F_{x}$ (figure 3). As may be seen in the figure there is a significant difference between $L_{0}$ and $L_{r}$ and this change will directly affect the CDPR platform positioning.

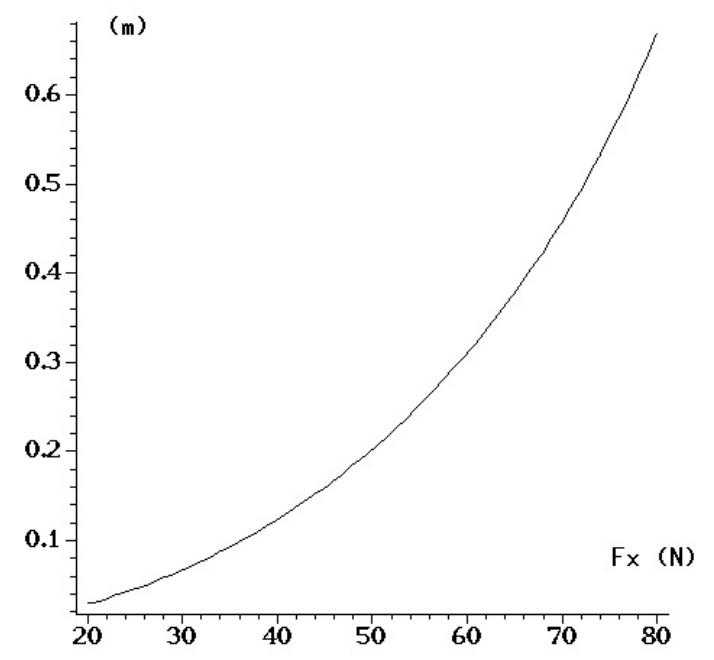

Figure 3: Difference in meter between the cable length and the distance between the attachment points $A, B$ as function for $F_{x}(\mathrm{~N})$ for a total tension of $100 \mathrm{~N}$ and a cable length at rest of 50 meters. This figure clearly shows that the sagging effect induces a large difference between the measured cable length and the distance between $A, B$ as soon as $F_{x}$ increases.

As may be seen on the figure the cable deformation induces a significant 
difference between the distance between $A, B$ and the cable length. Neglecting these effects will introduce error in the kinematic analysis of CDPR.

Another interesting property of the Irvine equations is that when $E \rightarrow$ $\infty, \mu \rightarrow 0$, then they provide exactly the ideal cable model [15]. This point has been used in [15], [16] to propose algorithms for solving the inverse and direct kinematics of CDPR. The main idea of these algorithms is to start with the solutions of these problems for ideal cables (ie. $E=\infty, \mu=0$ ) and for each of these solutions to incrementally move $E, \mu$ toward their real values, using a guaranteed Newton scheme to find the new solution at each step. Another interest of the Irvine equations is that they provide a very compact description of the cable effect with very few physical parameters, namely $E, \mu$. The $\mu$ parameter may easily been estimated accurately, while $E$ is more difficult to estimate and is time-varying. However we believe that an auto-calibration of $E$ (which is outside the scope of this paper) based on additional sensors giving information on the state of the robot (see [17] for example) is doable. On the other hand using the lumped-mass model [14] will require much more parameters (number and location of the nodes, mass and spring stiffness) that are difficult to estimate and has never been proven experimentally for CDPR. Furthermore the low number of parameters in the Irvine equations allows one to manage uncertain values with interval analysis, while increasing the number of parameters will make this task much more difficult.

Equations $(1,2)$ have as variables $x_{b}, z_{b}, L_{0}, F_{x}, F_{z}$. In the remaining sections of this paper we will consider that a combination of these variables have known values and we will determine closed-form solution for the remaining variables or univariate polynomials in one of the variable for which the maximal number of roots will be established. Some works have addressed this topic especially assuming that 3 of these 5 variables have a fixed value and establishing 2 equations for the remaining variables[18] whose solution is determined numerically. Our first contribution will be to establish new relationships between the variables that have not, to the best of the author knowledge, been proposed before.

Our second contribution addresses the use of these new relationships for the solving of the inverse and direct kinematics (IK and DK) of CDPR for which the cable model will obviously play an essential role. Cable model also influences the static analysis whose purpose is to determine the tension in the cables [19].

For the IK $n$ cables are attached to a rigid body in a known pose (hence 
the cable plane and the $x_{b}, z_{b}$ of each cable are known) with the purpose of determining $L_{0}$. One may notice that $(1,2)$ in their current form do not provide a closed-form for $L_{0}$. Hence solving the IK requires solving an equation system in $2 n$ equations $(1,2)$ with $3 n$ unknowns and of the mechanical equilibrium of the platform that imposes 6 additional equations. If $n=6$ we end-up with a square system of equations [20],[21],[22], To solve the IK authors have used optimization or have assumed that the solution is sufficiently close to the rigid leg case which is therefore used as initial guess for a solving based on the Newton scheme. However these methods cannot with certainty find all the solutions (as it has already be proven that the IK may have multiple solutions).

In the DK problem the $n L_{0}$ are known and the platform poses have to be determined. The unknowns are here the 6 parameters that define the platform pose and the $2 n F_{x}, F_{z}$ while the constraints are the $2 n$ equations $(1,2)$ and the 6 equations of the mechanical equilibrium, so that we have always a square system that has usually multiple solutions. Note however that the DK assumes the measurement of $L_{0}$ while current systems provided the stressed length so that corrective steps should theoretically be applied. As equations $(1,2)$ are not algebraic we cannot use methods such as elimination or Groebner basis that may provide all solutions for the DK and IK. Continuation method [23] are an option but requires a starting point. A natural starting point is to consider the cables as ideal and using the solutions of the IK and DK. However singularity are crossed during the continuation and are difficult to manage $[16,24]$. It was recently proposed to solve the DK by looking at the minima of the potential energy of CDPR [25] but finding numerically all these minima is tedious and uncertain.

We have addressed the IK and DK solving issues in previous publications, using as solving method an interval analysis-based approach that is guaranteed to provide all solutions assuming that the unknowns are bounded [26, 27] and is able to manage different cable models. The next section presents the principle of solving based on interval analysis and explains how the additional relationships we will provide in this paper may speed-up the solving. Note that this paper is an extended version of the conference paper [28] with new results and examples that illustrate the use of this work for the kinematic analysis of CDPR. 


\section{Interval analysis}

Interval analysis is based on interval evaluation of a function $f$ in the unknowns $\left\{x_{1}, x_{2}, \ldots x_{n}\right\}$ that are supposed to be bounded i.e. for each $x_{i}$ we have $x_{i} \in\left[\underline{x_{i}}, \overline{x_{i}}\right]$ where $\underline{x_{i}}, \overline{x_{i}}$ are respectively the lower and upper bound for $x_{i}$. Such bounds define a box in the $n$-dimensional space of the unknowns. Being given such a box $\mathcal{B}$ the interval evaluation $\hat{f}$ of $f$ over $\mathcal{B}$ is an interval $[\underline{f}, \bar{f}]$ such that for any point $X$ in $\mathcal{B}$ we have $f \leq f(X) \leq \bar{f}$. In other words $f$ is either equal to or a minorant of the minimum $f_{\min }$ of $f$ over $\mathcal{B}$ while $\overline{\bar{f}}$ is equal to or a majorant of the maximum $f_{\max }$ of $f$ over $\mathcal{B}$. The interval evaluation of $f$ is relatively easy to obtain if $f$ is expressed in terms of classical mathematical functions using the natural evaluation which basically consist in replacing the operators by interval equivalents. For example interval evaluation of the Irvine equations may be obtained by natural evaluation. A property of interval analysis is that two mathematically equivalent forms of $f$ may have different interval evaluations. For example $f^{1}=x^{2}+2 x+1$ and $f^{2}=(x+1)^{2}$ are equivalent but $\hat{f}^{2}$ will be tight with only one occurrence of $x$ while $f^{1}$ will not if $\underline{x}<0$. A solving algorithm may then be designed just by discarding boxes for which we have for any equation in the set either $\underline{f}>0$ or $\bar{f}<0$ as this shows that there is no solution of this equation for the variable in the box. Otherwise the box is bisected: one variable is chosen and its range is bisected at its mid -point so that two new boxes are created differing just by this variable range. It is not needed to bisect a box until it is reduced to a point as sophisticated methods $[29,30,31,32]$ allow to determine if a single root is present in a box (provided that it is small enough) and provide a numerical method to calculate this root.

However the efficiency of interval algorithms is drastically dependent upon the tightness of the interval evaluation: the closer $\underline{f}, \bar{f}$ are to $f_{\min }, f_{\max }$, the faster will be the algorithm. An interval evaluation will be denoted tight if $\hat{f}=\left[f_{\text {min }}, f_{\text {max }}\right]$. But the natural evaluation may lead to large under or overestimation of the minimum and maximum as soon as there are multiple occurrences of the unknowns in $f$ (it may be proven that if there is only a single occurrence of each unknown in $f$, then $\hat{f}$ is tight, up to round-off errors). The tightness will improve when the widths of the intervals for the unknowns decrease but an efficient way to improve the tightness of the evaluation is to consider the derivatives of $f$ and their own interval evaluation. Let $f_{i}$ be the derivative of $f$ with respect to $x_{i}$ and let $\left[\underline{f_{i}}, \overline{f_{i}}\right]$ be its interval 
evaluation over $\mathcal{B}$. If $f_{i}>0$ or $\overline{f_{i}}<0$, then $f$ is monotonic with respect to $x_{i}$. Consequently $\hat{f}$ may be obtained as $\left[\operatorname{Min} \hat{f}\left(\mathcal{B}_{i}\right), \operatorname{Max} \hat{f}\left(\mathcal{B}_{i}\right)\right]$ where $\mathcal{B}_{i}$ are the boxes that are derived from $\mathcal{B}$ with $x_{i}$ set to $\underline{x_{i}}$ or $\overline{x_{i}}$. Note that this process has to be applied recursively. Indeed assume that there is a $j>1$ such that $f$ is monotonic with respect to $x_{j}$ (implying that $\hat{f}$ will be obtained using $\mathcal{B}_{j}$ ), while for $i<j$ this was not the case. But for $i<j$ the monoticity has been evaluated using $\mathcal{B}$ and as we are now using the tighter $\mathcal{B}_{j}$ the monoticity test may give another result. Using this process we may tighten the interval evaluation of $f$ up to the point where $\hat{f}=\left[f_{\text {min }}, f_{\text {max }}\right]$ if $f$ is such that all $f_{i}, i \in[1, n]$ are positive or negative. Besides the use of derivatives another method is usually efficient to decrease the computation time. Let us assume that an equation may be rewritten as $H\left(x_{i}\right)=G\left(x_{1}, \ldots x_{i-1}, x_{i+1} \ldots x_{n}\right)$ where $H$ is an invertible function of $x_{i}$. Let $\hat{G}=[\underline{G}, \bar{G}]$ be the interval evaluation of $G$ for a given box. The range $\hat{x}_{i}$ for the unknown $x_{i}$ is updated by $\hat{x}_{i} \cap H^{-1}(\hat{G})$ and the box is discarded if this intersection is empty. We may also sharpen the range for $x_{i}$ by computing $\left[x_{i}, x_{i}+\epsilon\right] \cap \hat{G}$ where $\epsilon$ is a small value: if this intersection is empty then the lower bound for $x_{i}$ becomes $\underline{x_{i}}+\epsilon$ and we may repeat the process. A similar procedure may be used for the upper bound.

One difficulty of interval analysis is determining the right combination of heuristics that leads to the best computation time being given that this heuristics may reduce the computation time of the basic interval analysis algorithm from an almost intractable value to a few seconds.

Our second contribution is to present in the next sections some interesting properties of the Irvine equations that can be used for analysis or solving purposes.

\section{Properties of the Irvine equations}

A preliminary property will play an important role: we have assumed that $B$ has an altitude that is equal or lower to the one of $A$ with the direct consequence that $F_{z} \leq \mu g L_{0} / 2\left(F_{z}\right.$ must be lower than this value as soon as $B$ is lower than $A$ ).

\subsection{Derivatives of the Irvine equations}

The sign of the derivatives of the Irvine equations may be obtained with interval evaluation but it is interesting to determine beforehand if they may be inherently monotonic. 
Under assumption 1 we may establish the sign of derivatives of equations $(1),(2)$ that will be presented without proof as they are trivial. We have

$$
\frac{\partial z_{b}}{\partial L_{0}}<0 \quad \frac{\partial z_{b}}{\partial F_{z}}>0 \quad \frac{\partial z_{b}}{\partial F_{x}}>0
$$

As all derivatives of $z_{b}$ have a constant sign, then its interval evaluation for interval values for $F_{x}, F_{z}, L_{0}$ will always be tight and can be computed efficiently using only floating point operators. This may have an impact on the IK solving in which $z_{b}$ has a fixed value: If $\hat{z_{b}} \cap z_{b}=\emptyset$, then (2) has no solution for the current $F_{x}, F_{z}, L_{0}$ box. We have also:

$$
\frac{\partial x_{b}}{\partial L_{0}}>0 \quad \frac{\partial x_{b}}{\partial F_{z}}>0 \quad \frac{\partial x_{b}}{\partial F_{x}}>0
$$

It may also be interesting to consider the distance $D=x_{b}^{2}+z_{b}^{2}$ between $A$ and $B$. We have $\partial D / \partial L_{0}>0$ but no general monotonicity can be obtained with respect to $F_{x}, F_{z}$.

Let $F_{x}, F_{z}, L_{0}$ being bounded i.e. $F_{x} \in\left[\underline{F_{x}}, \overline{F_{x}}\right], F_{z} \in\left[\underline{F_{z}}, \overline{F_{z}}\right], L_{0} \in$ $\left[\underline{L_{0}}, \overline{L_{0}}\right]$. Let us assume that $z_{b}$ is fixed and consider the equation $\bar{f}\left(L_{0}, F_{z}, F_{x}\right)$ $\overline{z_{b}}=0$. Using the implicit value theorem it may be shown that the solution of this equation satisfies

$$
\frac{\partial F_{x}}{\partial L_{0}}>0 \quad \frac{\partial F_{x}}{\partial F_{z}}<0
$$

so that $F_{x}$ is restricted to lie in the interval $\left[\underline{F_{x}^{\prime}}, \overline{F_{x}^{\prime}}\right]$ where $\underline{F_{x}^{\prime}}$ is the solution of (2) obtained for $L_{0}=\overline{L_{0}}, F_{z}=F_{z}$ and $\overline{F_{x}^{\prime}}$ is the solution of (2) obtained for $L_{0}=\underline{L_{0}}, F_{z}=\overline{F_{z}}$. The range for $\overline{F_{x}}$ may therefore be calculated as $\left[\underline{F_{x}}, \overline{F_{x}}\right] \cap$ $\left[F_{x}^{\prime}, \overline{F_{x}^{\prime}}\right]$ and the equation has no solution if this intersection is empty. More generally if we consider (2) when 2 of the unknowns are fixed and denotes its solution by $S$ in the last unknown we get $\left[\underline{L_{0}^{\prime}}, \overline{L_{0}^{\prime}}\right]=\left[S\left(\overline{F_{x}}, \overline{F_{z}}\right), S\left(\underline{F_{x}}, \underline{F_{z}}\right)\right]$ and $\left[\underline{F_{z}^{\prime}}, \overline{F_{z}^{\prime}}\right]=\left[\left(S\left(\underline{F_{x}}, \overline{L_{0}}\right), S\left(\overline{F_{x}}, \underline{L_{0}}\right)\right]\right.$.

\subsection{New forms for the Irvine equation}

We present in this section various new relationships between the quantities appearing in the Irvine equations. They are usually expressed in a semiexplicit form $H(X)=G(Y)$ where $X$ is an element of the set $\left\{x_{b}, z_{b}, F_{x}, F_{z}, L_{0}\right\}$ while $Y$ is the complementary of $X$ with respect to this set. The function $H$ will be invertible and its definition may impose some constraint on $G$. In the numerical examples we will set $E=1^{11} \mathrm{~N} / \mathrm{m}^{2}, \mu=0.346 \mathrm{~kg} / \mathrm{m}$ and the cable diameter to $6 \mathrm{~mm}$. 


\subsubsection{Using the $z_{b}$ equation}

$\frac{F_{x} \text { as function of } z_{b}, F_{z}, L_{0}}{\text { Let }}$.

$a^{2}=F_{x}^{2}+F_{z}^{2} \quad b^{2}=F_{x}^{2}+\left(F_{z}-\mu g L_{0}\right)^{2} \quad a^{2}-b^{2}=\mu g L_{0}\left(2 F_{z}-\mu g L_{0}\right)<0$

then $z_{b}$ may be written as

$$
z_{b}=\left(\frac{(a-b)}{\mu g}\right)\left(\frac{(a+b)}{2 E A_{0}}+1\right)=\frac{L_{0}\left(2 F_{z}-\mu g L_{0}\right)}{2 E A_{0}}+\frac{a-b}{\mu g}
$$

Let us assume now that $z_{b}, F_{z}, L_{0}$ are given so that (2) has only $F_{x}$ as unknown. Our objective is to get an expression of this unknown. Let us define

$$
a^{2}=F_{x}^{2}+F_{z}^{2} \quad b^{2}=F_{x}^{2}+\left(F_{z}-\mu g L_{0}\right)^{2} \quad U=\frac{L_{0}\left(F_{z}-\mu g L_{0} / 2\right)}{E A_{0}}-z_{b}
$$

so that equation (2) may be written as

$$
U+\frac{(a-b)}{\mu g}=0
$$

We have also

$$
a^{2}-b^{2}=2 F_{z} \mu g L_{0}-\left(\mu g L_{0}\right)^{2}=V=(a+b)(a-b)=(a+b)(-U \mu g)
$$

from which we get

$$
b=-\frac{V}{U \mu g}-a
$$

Reporting $b$ in (6) leads to

$$
2 a=-U \mu g-\frac{V}{U \mu g}=W
$$

Note that $U, V$ are not function of $F_{x}$ so that $W$ is expressed only as a function of $F_{z}, L_{0}$. As $a^{2}=(W / 2)^{2}=F_{x}^{2}+F_{z}^{2}$ we get

$$
F_{x}^{2}=(W / 2)^{2}-F_{z}^{2}
$$

where the right-hand term is a function of $F_{z}, L_{0}$ only. This equation provides $F_{x}$ if $z_{b}, L_{0}, F_{z}$ are fixed but also imposes a constraint on $(W / 2)^{2}-F_{z}^{2}$ that should be positive. 
Let's assume that $F_{z}$ has an interval value and consider $P=F_{x}^{2}=$ $(W / 2)^{2}-F_{z}^{2}$ that is positive. The polynomial $P$ is of degree 4 in $F_{z}$ and factors out in 4 terms that are linear in $F_{z}$. The roots of $P$ are

$$
s_{1}=\frac{\mu g L_{0}}{2}+\frac{\mu g A_{0} E z_{b}}{2 A_{0} E+\mu g L_{0}} \quad s_{2}=\frac{\mu g L_{0}}{2}+\left(z_{b}-L_{0}\right) \frac{A_{0} E}{L_{0}}
$$

and

$$
s_{3}=\frac{\mu g L_{0}}{2}+\left(z_{b}+L_{0}\right) \frac{A_{0} E}{L_{0}} \quad s_{4}=\frac{\mu g L_{0}}{2}-\frac{\mu g A_{0} E z_{b}}{2 A_{0} E-\mu g L_{0}}
$$

If we assume $2 A_{0} E>\mu g L_{0}$ then the roots in $F_{z}$ are ordered as $s_{2}, s_{1}(<$ $\left.\mu g L_{0} / 2\right), s_{4}\left(>\mu g L_{0} / 2\right), s_{3}$ and $P$ will be positive if $F_{z} \in\left[s_{2}, s_{1}\right]$. If $2 A_{0} E<$ $\mu g L_{0}$ then the roots are ordered as $s_{2}, s_{4}\left(<\mu g L_{0} / 2\right), s_{1}\left(<\mu g L_{0} / 2\right), s_{3}$. Therefore there are 2 possible ranges for $F_{z}$ leading to a positive $P$ : $\left[s_{2}, s_{4}\right]$, $\left[s_{1}, \mu g L_{0} / 2\right]$. The previously determined range for $F_{z}$ may be used to update the range of several variables in an interval analysis based algorithm.

Numerical example: Consider the case where $L_{0} \in[50,50.4], F_{x} \in$ $[100,130], F_{z} \in[-130,-100]$. The interval estimation of $z_{b}$ based on equation (2) is $[-54.954,-30.586]$. Using equation (8) leads to the range $[0,286.34]$ for $F_{x}$. But if we restrict $z_{b}$ to the range $[-54.954,-49]$ or $[-37,-30.586]$ the range for $F_{x}$ as deduced from (8) does not have an intersection with the range $[100,130]$. Therefore we can claim that $z_{b}$ is restricted to $\left.]-49,-37\right]$ : this interval is 7 times smaller than the one that has initially been obtained.

$L_{0}$ as function of $z_{b}, F_{z}, F_{x}$.

We are now interested in determining $L_{0}$ when $F_{x}, F_{z}, z_{b}$ are fixed. Let $U_{1}=$ $\sqrt{F_{x}^{2}+F_{z}^{2}}, U_{2}=\mu g F_{z} / E A_{0}, U_{3}=(\mu g)^{2} /\left(2 E A_{0}\right)$ and $U_{4}=-\mu g z_{b}+U_{1}$. Equation (2) may be written as

$$
U_{2} L_{0}-U_{3} L_{0}^{2}+U_{4}=\sqrt{F_{x}^{2}+\left(F_{z}-\mu g L_{0}\right)^{2}}
$$

Squaring the previous equation leads to

$$
P_{s}=\left(U_{2} L_{0}-U_{3} L_{0}^{2}+U_{4}\right)^{2}-\left(F_{x}^{2}+\left(F_{z}-\mu g L_{0}\right)^{2}\right)=0
$$

As $U_{1}, U_{2}, U_{3}, U_{4}$ are not functions of $L_{0}>0$ this equation is a fourth order polynomial in $L_{0}$. Using the Sturm sequences it is possible to show that $P_{s}$ has only 2 roots for $L_{0}$ in the range $[0, \infty]$. One of these root leads to

$$
U_{2} L_{0}-U_{3} L_{0}^{2}+U_{4}=-\sqrt{F_{x}^{2}+\left(F_{z}-\mu g L_{0}\right)^{2}}
$$


that is not compatible with equation (9). Therefore solving $P_{s}$ (whose roots may be obtained in analytical form but cannot be displayed here for lack of space) leads to a single solution for $L_{0}$. Note that for an interval-based algorithm we will first compute an interval evaluation of $P_{s}$ to determine if it may have a zero and it is not necessary to use the analytical form of the roots to obtain bounds for $L_{0}$ using only the interval evaluation of the polynomial coefficients $[33,34]$. As we may have a tight range for $L_{0}$ (e.g. in the DK problem) another possibility is to look at the Sturm sequences (or at the simpler Budan-Fourier sequence) of the polynomial for this range. whose elements are functions of $F_{x}, F_{z}$. If there is no root to the polynomial (10), then the number of sign changes for $\overline{L_{0}}$ minus the number of sign changes for $\underline{L_{0}}$ should be equal to 0 , thereby inducing inequality constraints on $F_{x}, F_{z}$ that may possibly allow one to tighten the ranges for these variables.

Numerical example: we assume that $L_{0}$ is measured and lie in the range $[50,50.03]$ meter . A sensor on the platform allows to get an estimation of $z_{b}$ as $-42.3922 \pm 0.05$ meter. The force $F_{x}$ is estimated to be in the range $[100,110]$ while $F_{z}$ is in the range $[-130,-110]$. An analysis of the BudanFourier sequence for $L_{0}=50$ and $L_{0}=50.03$ shows that the polynomial $P_{s}$ has a root in the $L_{0}$ range only if $P_{s}(50) \times P_{s}(50.03)<0$. An analysis of the derivatives of $P_{s}$ with respect to $F_{x}, F_{z}$ shows that they are positive. As for $F_{x}=110, F_{z}=-110$ we have $P_{s}([50,50.03])<0$ we deduce that the polynomial $P_{s}$ has no root whatever the values of $F_{x}, F_{z}$ in their respective range are.

$F_{z}$ as function of $z_{b}, F_{x}, L_{0}$.

$\overline{\text { We consider determining } F_{z}}$ for given $L_{0}, F_{x}, z_{b}$. Equation (8) is a 4 th order polynomial $Q$ in $F_{z}$ with the constraint that $W>0$. Using Budan-Fourier theorem [33] it is possible to show that $Q$ has 0 or 2 roots in the range ] $-\infty, \mu g L_{0} / 2$ ] but only one these roots will lead to a positive $W>0$. The analysis of the sign of $W$ is complex but it may be shown that if $E A_{0} \gg \mu g L_{0}$, then $F_{z}$ must belong to the range $\left[\mu g L_{0} / 2+E A_{0} z_{b} / L_{0}, \mu g L_{0} / 2-\mu g z_{b}^{2} /\left(2 L_{0}\right)\right]$.

\subsubsection{Using the $x_{b}$ equation}

$F_{z}$ as function of $x_{b}, F_{x}, L_{0}$.

We consider the calculation of $F_{z}$ being given $F_{x}, L_{0}, x_{b}$. We define

$$
u=\frac{F_{z}}{F_{x}} \quad v=\frac{F_{z}-\mu g L_{0}}{F_{x}}
$$


so that equation (1) may be written as

$$
\left(\frac{x_{b}}{F_{x}}-\frac{L_{0}}{E A_{0}}\right) \mu g=\sinh ^{-1}(u)-\sinh ^{-1}(v)
$$

We define $H_{1}=x_{b} / F_{x}-L_{0} /\left(E A_{0}\right)$ and we use the identity

$$
\sinh ^{-1}(u)-\sinh ^{-1}(v)=\sinh ^{-1}\left(u \sqrt{1+v^{2}}-v \sqrt{1+u^{2}}\right)
$$

Using the the hyperbolic sine of both terms of equation (11) we obtain:

$$
H\left(x_{b}, L_{0}, F_{x}\right)=\sinh \left(H_{1} \mu g\right)=u \sqrt{1+v^{2}}-v \sqrt{1+u^{2}}
$$

We have already defined $a^{2}=F_{x}^{2}+F_{z}^{2}, b^{2}=F_{x}^{2}+\left(F_{z}-\mu g L_{0}\right)^{2}$ so that $a^{2}=F_{x}^{2}\left(1+u^{2}\right)$ and $b^{2}=F_{x}^{2}\left(1+v^{2}\right)$. Equation (12) may therefore be written as:

$$
F_{x} H\left(x_{b}, L_{0}, F_{x}\right)=u b-v a
$$

Note that the left-hand term of this equation is not a function of $F_{z}$. Let us define $W=F_{x} H\left(x_{b}, L_{0}, F_{x}\right)$ and $u_{1}, u_{2}$ such that $1+u^{2}=u_{1}^{2}, 1+v^{2}=u_{2}^{2}$ so that

$$
W=u b-v a=F_{z} u_{2}-u_{1}\left(F_{z}-\mu g L_{0}\right)
$$

We have also $u_{1}^{2}-u_{2}^{2}=u^{2}-v^{2}$ so that

$$
u_{1}^{2}-u_{2}^{2}=\left(2 F_{z} \mu g L_{0}-\left(\mu g L_{0}\right)^{2}\right) / F_{x}^{2}
$$

Solving (14) for $u_{2}$ and reporting it in (15) leads to an equation in $u_{1}, F_{x}, F_{z}, L_{0}, W$ which is of second order in $u_{1}$. Substituting $u_{1}^{2}=\left(F_{x}^{2}+F_{z}^{2}\right) / F_{x}^{2}$ in this equation lead to a linear equation in $u_{1}$. This equation is solved in $u_{1}$ and the result is reported in $u_{1}^{2}=\left(F_{x}^{2}+F_{z}^{2}\right) / F_{x}^{2}$ that becomes a polynomial of order 4 in $F_{z}$ whose coefficients are functions of $F_{x}, L_{0}, x_{b}$

\section{$\underline{L_{0} \text { as function of } x_{b}, F_{x}, F_{z}}$.}

Consider equation $x_{b}-x_{b}^{s}$ where $x_{b}^{s}$ is a desired value for $x_{b}$ and $x_{b}$ is provided by equation (1) and $F_{x}, F_{z}$ are given. This equation may be written as

$$
F\left(L_{0}\right)=u 2 L_{0}-u_{3} \sinh ^{-1}\left(u_{4}+u_{5} L_{0}\right)-u_{1}=0
$$

with $-u_{1}=x_{b}^{s} / F_{x}-\sinh ^{-1}\left(F_{z} / F_{x}\right) /(\mu g), u_{2}=1 /\left(E A_{0}\right)>0, u_{3}=1 /(\mu g)>$ $0, u_{4}=F_{z} / F x, u_{5}--\mu g / F_{x}<0$. Although it seems difficult to derive 
a closed-form for the root in $L_{0}$ of this equation, it appears that $F$ has interesting properties. Indeed the derivative of $F$ with respect to $L_{0}$ is strictly positive, while $F(0)=-x_{b}^{s} / F_{x}<0$, and consequently there is a single root in $L_{0}$ for $F=0$. Furthermore as the term $u_{2} L_{0}$ is positive if $-u_{3} \sinh ^{-1}\left(u_{4}+\right.$ $\left.u_{5} L_{0}\right)>u_{1}$, then $F>0$. Further manipulation of this inequality leads to $F>0$ if $L_{0}>\left|u_{5}\right| \sinh ^{-1}\left(u_{1} / u_{3}\right) / u_{4}=L_{0}^{M}$. Hence $L_{0}^{M}$ is an upper bound for the root of $F=0$. A simple dichotomy procedure allows one to obtain quickly an estimation of the root. At each step of the dichotomy we check if the Kantorovitch theorem conditions [35] hold for the current estimation of the root so that the Newton scheme will converge to the solution. Such a procedure leads to a very fast determination of the root, that may be obtained with an arbitrary accuracy. If $F_{x}, F_{z}$ are provided as intervals, then rewriting $F$ as $L_{0}=\left(u_{3} \sinh ^{-1}\left(u_{4}+u_{5} L_{0}\right)+u_{1}\right) / u_{2}$ may be useful to decrease the range for $L_{0}$.

Numerical example: we set $F_{x}=1, F_{z}=-10, x_{b}^{s}=0.5$. We obtain $L_{0}^{M}=18.571$. For $L_{0}^{m} / 2$ we get $F<0$ so that we set $L_{0}=\left(L_{0}^{M} / 2+L_{0}^{M}\right) / 2=$ 13.92855. For that value the Kantorovitch conditions hold and the Newton scheme provide the solution $L_{0}=13.1732$.

$\underline{F_{x} \text { as function of } x_{b}, L_{0}, F_{z}}$.

Consider equation $x_{b}-x_{b}^{s}$ where $x_{b}^{s}$ is a desired value for $x_{b}$ and $x_{b}$ is provided by equation (1) and $L_{0}, F_{z}$ are given. It seems difficult to derive a closed-form for the root in $F_{x}$ of this equation. But we have shown in section 3.1 that $\partial x_{b} / \partial F_{x}$ is positive. As the limit of $x_{b}$ when $F_{x}$ goes to 0 is 0 while its limits when $F_{x} \rightarrow \infty$ is $L_{0}$. Hence this equation has a single root in $F_{x}$.

We note also that $x_{b}=F_{x} L_{0} /\left(E A_{0}\right)+\alpha$ where $\alpha$ is positive. Consequently we have $F_{x} L_{0} /\left(E A_{0}\right)<x_{b}$ or $F_{x}<E A_{0} x_{b} / L_{0}$, this inequality providing an upper bound for the root of $F_{x}$. Hence a dichotomy process, mixed with the use of the Newton method as described in the previous section, will provide efficiently the root.

\subsubsection{Using the $x_{b}$ and $z_{b}$ equations}

$\underline{F_{z} \text { as function of } x_{b}, z_{b}, F_{x}, L_{0}}$. 
We proceed along the same direction than the calculation of $F_{z}$ described in section 3.2.2 using the equation

$$
F_{x} H\left(x_{b}, L_{0}, F_{x}\right)=u b-v a
$$

We have already established in section 3.2.1 the values of $a, b$ as functions of $z_{b}, L_{0}, F_{z}$ while $u, v$ are functions of $F_{x}, L_{0}, F_{z}$. Hence the right-hand term of (17) is a function of $z_{b}, L_{0}, F_{x}, F_{z}$. This function is a third order polynomial $P_{3}$ in $F_{z}$. Using the Sturm sequence [33] and the constraint $a>0$ it is possible to show that $P_{3}$ has a single real root in the range ] $-\infty, \mu g L_{0} / 2$ ].

Numerical example: we assume $x_{b} \in[24.65,24.8], z_{b} \in[-43.5,-43]$, $F_{x} \in[90,100], F_{z} \in[-90,-85]$ and $L_{0} \in[49,49.02]$. The number of sign changes of the Budan-Fourier sequence for $F_{z}=-90$ and for $F_{z}=-85$ are both one so that $P_{3}$ has no root in its interval.

$z b$ as a function of $x_{b}, F_{x}, F_{z}, L_{0}$.

Equation (2) provides a mean of calculating $z_{b}$ when $F_{x}, F_{z}, L_{0}$ are known but does not involve $x_{b}$ and we provide here another form that involves $x_{b}$. Using the notation and result of section 3.2 .3 we get:

$$
z_{b}=\frac{L_{0}}{F_{z}}\left(\sqrt{F_{x}^{2}+F_{z}^{2}}-\frac{F_{x}^{2}}{\mu g L_{0}} \sinh \left(\mu g\left(\frac{x_{b}}{F_{x}}-\frac{L_{0}}{E A_{0}}\right)\right)\right)+\frac{L_{0}\left(F_{x}-\mu g L_{0} / 2\right)}{E A_{0}}
$$

Note that we may also obtain a bound on the cable tension $\sqrt{F_{x}^{2}+F_{z}^{2}}$ at $B$ as

$$
\sqrt{F_{x}^{2}+F_{z}^{2}}=\frac{F_{x}^{2}}{\mu g L_{0}} \sinh \left(\mu g\left(\frac{x_{b}}{F_{x}}-\frac{L_{0}}{E A_{0}}\right)\right)+F_{z}\left(\frac{z_{b}}{L_{0}}+\frac{\mu g L_{0}}{2 E A_{0}}-\frac{F_{z}}{E A_{0}}\right)
$$

\subsection{Using the cable tangents}

Sensors may provide a relatively accurate measurement of the cable tangents $v=\left(F_{z}-\mu g L_{0}\right) / F_{x}$ at $A$ and $u=F_{z} / F_{x}$ at $B$ [17]. Under the assumption that $u, v$ are known we get

$$
F_{x}=\frac{\mu g L_{0}}{(u-v)} \quad F_{z}=u F_{x} \quad F_{x}^{2}+F_{z}^{2}=\left(\frac{\mu g L_{0}}{(u-v)}\right)^{2}\left(1+u^{2}\right)
$$

A trivial transformation of (2) leads to:

$$
\mu g L_{0}^{2}(u+v)+2 A_{0} E L_{0}\left(\sqrt{u^{2}+1}-\sqrt{v^{2}+1}\right)+2 z_{b} E A_{0}(v-u)=0
$$


which is a quadratic polynomial in $L_{0}$ whose coefficients are functions of $u, v, z_{b}$. It is easy to show that this polynomial has a single positive root. Now equation (1) may be written as

$$
F_{x}\left(\frac{L_{0}}{E A_{0}}+\frac{\left(\sinh ^{-1}(u)-\sinh ^{-1}(v)\right)}{\mu g}\right)-x_{b}=0
$$

As we have $F_{x}=\mu g L_{0} /(u-v)$ this equation may be transformed in a second order polynomial in $L_{0}$ whose coefficients are functions of $u, v, x_{b}$. Here again it is easy to show that this polynomial has at most one positive root.

As $F_{z}=u F_{x}$ and $L_{0}=\left(F_{x}(u-v)\right) /(\mu g)$ equations (11), (22) are polynomials in $F_{x}$ with coefficients that are functions of $u, v$. The resultant of these equations in $F_{x}$ establishes a polynomial relationship between $x_{b}, z_{b}$ which is a quadric, more precisely a parabola which is written as

$$
\left(A x_{b}+C z_{b}\right)^{2}+D x_{b}+F z_{b}=0
$$

with

$$
\begin{gathered}
A=\sqrt{\mu g}(u-v)(u+v) \quad C=-2 \sqrt{\mu g}(u-v) \\
D=2 E A_{0}(u-v)\left(R_{1} \mu g(u+v)-2 R_{2}\right) R_{2} \\
F=-2 \mu g E A_{0}(u-v)\left(R_{1} \mu g(u+v)-2 R_{2}\right) R_{1} \\
R_{1}=\left(\sinh ^{-1}(u)-\sinh ^{-1}(v)\right) /(\mu g) \quad R_{2}=\sqrt{1+u^{2}}-\sqrt{1+v^{2}}
\end{gathered}
$$

Note that if $E A_{0} \gg \mu g L_{0}$, then $A, C$ are small and $D, F$ very large so that the parabola is very close to a line.

The measurements of $u, v \cdot L_{0}$ provide a direct estimation of $F_{x}=\mu g L_{0} /(u-$ $v$ ) and consequently of $F_{z}=u F_{x}$ and therefore of the cable tension at $B$ without any force sensor. As these measurements are uncertain (however with a bounded uncertainty) we will get a range for $x_{b}, z_{b}$ using equations $(1,2)$ that may possibly be sharpened using the parabola equation (23). These sharpened evaluations may possibly be used to sharpen either $L_{0}, u, v$ and/or $F_{x}, F_{z}$.

\subsection{Summary}

Each of the two Irvine equations involves 4 variables: $x_{b}$ (or $z_{b}$ ), $F_{x}, F_{z}, L_{0}$. Consequently each equation may be used to determine one of the variables if the other 3 variables are known. If both equations are used we have 5 variables. If 3 of them are known the Irvine equations become a system of 2 
equations in 2 unknowns and we may, theoretically, obtain these 2 unknowns as functions of the known variables. We have not been able to reach this goal (except for the trivial case where $x_{b}, z_{b}$ are the unknowns). However if we assume that 4 variables are known, then the Irvine equations are overconstrained and may be used to calculate the remaining unknown. Table 1 summarizes the obtained result. In this table the unknown are presented as $F_{x}, F_{z}, L_{0}, x_{b}, z_{b}$. For one unknown there are several columns. Each of this column is either empty or has a $\bullet$ or a $\div$ symbol. A column with only indicates that the unknown can be calculated in closed-form if the indicated variables are set. A column with only $\div$ indicates that the unknown has not been obtained in closed-form but that an efficient numerical scheme can be designed to calculate the unknown. For example the first column for the unknown $F_{x}$ shows that $F_{x}$ can be calculated in closed-form if $F_{z}, L_{0}, z_{b}$ are known, while the second column shows that $F_{x}$ can be calculated numerically if $F_{z}, L_{0}, x_{b}$ are known.

\begin{tabular}{|c|c|c|c|c|c|c|c|c|c|c|}
\hline & \multicolumn{2}{|c|}{$F_{x}$} & \multicolumn{3}{|c|}{$\overline{F_{z}}$} & \multicolumn{2}{|c|}{$L_{0}$} & $x_{b}$ & \multicolumn{2}{|c|}{$z_{b}$} \\
\hline$\overline{\overline{F_{x}}}$ & & & $\bullet$ & $\bullet$ & $\bullet$ & $\bullet$ & $\div$ & $\bullet$ & $\bullet$ & $\bullet$ \\
\hline$F_{z}$ & $\bullet$ & $\div$ & & - & & $\bullet$ & $\div$ & $\bullet$ & $\bullet$ & $\bullet$ \\
\hline$L_{0}$ & $\bullet$ & $\div$ & $\bullet$ & $\bullet$ & $\bullet$ & & & $\bullet$ & $\bullet$ & $\bullet$ \\
\hline$x_{b}$ & & $\div$ & & $\bullet$ & $\bullet$ & & $\div$ & - & & $\bullet$ \\
\hline$z_{b}$ & $\bullet$ & & $\bullet$ & & $\bullet$ & $\bullet$ & & & & \\
\hline
\end{tabular}

Table 1: Summary of the result showing how the unknown indicated in the first row can be be obtained as function of the variables indicated in the first vertical column.

\section{Conclusion}

We have presented in this paper various results regarding the Irvine equations that may be useful both for analysis and solving of kinematic equations that rely on this cable model as they establish a more general view of the underlying structure of this model. We have shown that the proposed new forms of the Irvine equations may be useful to speed up the solving of the IK and DK of CDPR based on interval analysis (part of them have been implemented our CDPR IK and DK solver with a strong influence on the solving time for finding all solutions). But they may possibly also be used for alternate solving methods such as continuation. It must be reminded that 
all these solving methods are not intended to be used for real-time computation (i.e. within the sampling time of the CDPR controller) as generic and guaranteed Newton scheme exists for that purpose. Still open issues on the CDPR with sagging cables such as workspace and singularity analysis may benefit from this new approach to the Irvine equations.

\section{Acknowledgement:}

This paper has been partly supported by Agence Nationale de la Recherche (ANR), under grant ANR-18-CE10-0004-03 (CRAFT project)

\section{References}

[1] X. Tang, R. Yao, Dimensional design of the six-cable driven parallel manipulator of FAST, ASME J. of Mechanical Design 133 (11) ( November 2011) 111012-1/11.

[2] M. Gouttefarde, D. Nguyen, C. Baradat, Kinetostatics analysis of cabledriven parallel robots with consideration of sagging and pulleys, in: ARK, Ljulbjana, June 29- July 3, 2014, pp. 213-221.

[3] J.-P. Merlet, D. Daney, A portable, modular parallel wire crane for rescue operations, in: IEEE Int. Conf. on Robotics and Automation, Anchorage, May, 3-8, 2010, pp. 2834-2839.

[4] V. Schmidt, A. Pott, Investigating the effect of cable force on winch winding accuracy for cable-driven parallel robots, Proceedings of the Institution of Mechanical Engineers, Part K: Journal of Multi-body Dynamics 230 (3) (2016) 237-241.

[5] H. M. Irvine, Cable Structures, MIT Press, 1981.

[6] N. Riehl, et al., On the determination of cable characteristics for large dimension cable-driven parallel mechanisms, in: IEEE Int. Conf. on Robotics and Automation, Anchorage, May, 3-8, 2010, pp. 4709-4714.

[7] M. Ahmadi-Kashani, A. Bell, The analysis of cables subject to uniformly distributed loads, Eng. Struct. 10 (1988) 174-182.

[8] H. Hussein, M. Gouttefarde, F. Pierrot, Static modeling of sagging cables with flexural rigidity and shear forces, in: ARK, Bologna, July, $1-5,2018$. 
[9] S. Ghoreishi, et al., Analytical modeling of synthetic fiber rope. part II: a linear elastic model for $1+6$ fibrous structure, Int. J. of Solids and Structures (2007) 2943-2966.

[10] J. Piao, et al., Open-loop position control of a polymer cabledriven parallel robot via a viscoelastic cable model for high payload workspaces, Advances in Mechanical Engineering 9 (12).

[11] W. Samuel, et al., Synthetic mooring rope for marine renewable energy applications, Renewable energy 83 ( November 2015) 1268-1278.

[12] V. Schmidt, Modeling techniques and reliable real-time implementation of kinematics for cable-driven parallel robots using polymer fiber cables, Ph.D. thesis, Université Stuttgart ( June, 20, 2016).

[13] P. Tempel, F. Trautweing, A. Pott, Experimental identification of stressstrain material models of UHMWPE fiber cables for improving cable tension control strategies, in: ARK, Bologna, July, 1-5, 2018.

[14] J. Kamman, R. Huston, Multibody dynamics modeling of variable length cable systems, Multibody System Dynamics 5 (3) (2001) 211221.

[15] J.-P. Merlet, A new generic approach for the inverse kinematics of cabledriven parallel robot with 6 deformable cables, in: ARK, Grasse, June, 27-30, 2016.

[16] J.-P. Merlet, Preliminaries of a new approach for the direct kinematics of suspended cable-driven parallel robot with deformable cables, in: Eucomes, Nantes, September, 20-23, 2016.

[17] J.-P. Merlet, An experimental investigation of extra measurements for solving the direct kinematics of cable-driven parallel robots, in: IEEE Int. Conf. on Robotics and Automation, Brisbane, 2018.

[18] D. Papini, On shape control of cables under vertical static loads, Master's thesis, Lund University, Lund (2010).

[19] L. Hui, A giant sagging-cable-driven parallel robot of FAST telescope: its tension-feasible workspace of orientation and orientation planning, in: 14th IFToMM World Congress on the Theory of Machines and Mechanisms, Taipei, October, 27-30, 2015. 
[20] K. Kozak, et al., Static analysis of cable-driven manipulators with nonnegligible cable mass, IEEE Trans. on Robotics 22 (3) ( June 2006) 425-433.

[21] N. Riehl, et al., Effects of non-negligible cable mass on the static behavior of large workspace cable-driven parallel mechanisms, in: IEEE Int. Conf. on Robotics and Automation, Kobe, May, 14-16, 2009, pp. 2193-2198.

[22] D. Sridhar, R. Williams II, Kinematics and statics including cable sag for large cable suspended robots, Global Journal of Researches in Engineering: H Robotics \& Nano-Tec 17 (1).

[23] E. Allgower, Numerical continuation methods, Springer-Verlag, 1990.

[24] J.-P. Merlet, A generic numerical continuation scheme for solving the direct kinematics of cable-driven parallel robot with deformable cables, in: IEEE Int. Conf. on Intelligent Robots and Systems (IROS), Daejeon, October, 9-14, 2016.

[25] A. Pott, P. Tempel, A unified approach to forward kinematics for cabledriven parallel robots based on energy, in: ARK, Bologna, July, 1-5, 2018.

[26] J.-P. Merlet, The kinematics of cable-driven parallel robots with sagging cables: preliminary results, in: IEEE Int. Conf. on Robotics and Automation, Seattle, May, 26-30, 2015, pp. 1593-1598.

[27] J.-P. Merlet, On the inverse kinematics of cable-driven parallel robots with up to 6 sagging cables, in: IEEE Int. Conf. on Intelligent Robots and Systems (IROS), Hamburg, Germany, September 28- October 2, 2015, pp. 4536-4361.

[28] J.-P. Merlet, Some properties of the Irvine cable model and their use for the kinematic analysis of cable-driven parallel robots, in: EUCOMES, Aachen, September, 4-6, 2018.

[29] E. Hansen, Global optimization using interval analysis, Marcel Dekker, 2004. 
[30] L. Jaulin, M. Kieffer, O. Didrit, E. Walter, Applied Interval Analysis, Springer-Verlag, 2001.

[31] J.-P. Merlet, ALIAS: an interval analysis based library for solving and analyzing system of equations, in: SEA, Toulouse, June, 14-16, 2000.

[32] A. Neumaier, Interval methods for systems of equations, Cambridge University Press, 1990.

[33] P. Ciarlet, J.-L. Lions, Handbook of numerical analysis, 7 : solution of equations in Rn (part 3), North-Holland, 2000.

[34] J.-P. Merlet, Determination of the minimal and maximal real roots of parametric polynomials using interval analysis, in: 1st Int. Workshop on Global Constrained Optimization and Constraint Satisfaction (Cocos'02), Valbonne, October, 2-4, 2002, published in LNCS Volume 2861 / 2003,pp. 71-86.

[35] R. Tapia, The Kantorovitch theorem for Newton's method, American Mathematic Monthly 78 (1.ea) (1971) 389-392. 